Original Research Paper

\title{
A 10GH $_{Z}$ Low-Offset Dynamic Comparator for High-Speed and Lower-Power $\mathrm{ADC}_{S}$
}

\author{
Bakoune Pierre Hypolite, Wembe Tafo Evariste and Moukengue Imano Adolphe \\ Department of Physics, University of Douala, P.O. Box 24157 Douala, Cameroon
}

Article history

Received: $20-10-2018$

Revised: $17-11-2018$

Accepted: 04-05-2019

Corresponding Author: Wembe Tafo Evariste Department of Physics, University of Douala, P.O. Box 24157 Douala, Cameroon Email: twembee@yahoo.fr

\begin{abstract}
This paper proposed a design of low-voltage Dynamic Comparator using $90 \mathrm{~nm}$ PTM CMOS technology for high-speed and Lower-power Analog to Digital Converter (ADC) applications. The double tail structure is employed as based for design new comparator with positive feedback due to best behavior in low-voltage that allows low delay time; decreases the offset voltage and power dissipation. Simulation results are presented with sampling frequency of $10 \mathrm{GH}_{\mathrm{Z}}$. These results are also compared with earlier works interms of their delay time, power dissipation and offset voltage. The proposed comparator shows $5.7 \mathrm{mV}$ offset which is small when compared to other dynamic comparators and preamplifier based comparators.
\end{abstract}

Keywords: Dynamic, $90 \mathrm{~nm}$ PTM CMOS, Analog to Digital Converter (ADC), Double Tail Structure Positive Feedback

\section{Introduction}

The function of a comparator is to generate an output voltage whose value is high or low depending on the amplitude of the input. Fast and high accuracy comparators can be built using positive feedback circuits periodically enabled and reset by a clock. These synchronous comparators are systematically employed in the implementation of fast Analog to Digital Converters (ADC) and in many other analog systems such as Discriminators, peak detector.

Since comparator is one of the basic building blocks in most Analog to Digital Converters (ADCs). Comparators play a vital role in the overall performance of high-speed ADC converters. As decision-making circuits provide the interface between analog and digital signals, the accuracy, which is often determined by the input-referenced offset voltage, is critical for the resolution of high-performance ADCs. Dynamic comparators are widely used in high-speed ADCs because of its low power consumption and high speed.

In order to show the improvement results of our paper, the new Design based on double tail structure proposed, allows comparing with existing works the fast-speed, low-offset voltage, kickback noise and power dissipation, which are considerably improved. Schematic is drawn using the Ansys Electronics Desktop (Nexxim simulator). This paper is organized as follows. Section II describes the comparator architecture. Section III describes the analysis of the proposed dynamic latched comparator. Section IV provides schematics of various types of dynamic latched comparators. Simulation results using $90 \mathrm{~nm}$ PTM technology with $V_{D D}=1 \mathrm{~V}$ and their comparisons are presented in Section V. Finally, Section VI included Conclusion of the proposed work and future scope.

\section{Comparator Architectures and Previous Works}

Designed voltage comparator consists of three stages: Input stage, decision stage and output stage (Mongre and Gurjar, 2014) and the performance of any comparator is defined by the characteristic parameters such as offset voltage (Shaik and Rajesh, 2013), kickback noise (Pedro and Vital, 2006), clock frequency or speed (Iniewski, 2015), low-power consumption (Zbigniew, 2016), high resolution and random noise (Dastagiri and Hari Kishore, 2018). These parameters are mainly considered for design specifications.

Architecture of voltage comparators can be classified in to three types: Open-loop comparators (continuous time comparator), pre-amplifier based latch Comparator (latch with preamplifier) and fully Dynamic Latched Comparator (Shaik and Rajesh, 2013; Iniewski, 2015). However, due to its limited gain-bandwidth product and static power consumption, open-loop comparator are too 
slow for many applications. Pre-amplifier based latched comparator employ positive feedback to regenerate quickly voltage difference to full digital levels. It is a combination of Pre-amplifier and latch. Figure 1 shows CMOS differential pair loaded by cross-coupled transistors. The circuits operate between two states: In the reset mode, the switch $S_{R}$ its closed and positive feedback is disabled (Iniewski, 2015). However, the preamplifier based can be reduced latch offset voltage and also the kick-back noise, but suffer to limited gainbandwidth product static power consumption and reduction of the drain-to-source resistance $r_{d s}$ due to the continuous technology scaling.

The conventional dynamic comparator presented in Fig 2 is preferred to eliminate the static power consumption because this comparator dissipate power only during the regenerative phase and allows a faster operation (Wicht et al., 2004; Kale and Gamad, 2010). The operation of the comparator is as follows. During the reset phase when $C L K=0$ and $M_{R 3}$ is off, reset transistors $M_{R 1}$ and $M_{R 2}$ pull both output nodes $V_{\text {out } 1}$ and $V_{\text {out } 2}$ to $V_{D D}$ to define a start condition and to have a valid logical level during reset. In the comparison phase, when $C L K=V_{D D}$, transistors $M_{R 1}$ and $M_{R 2}$ are off and $\mathrm{M}_{\mathrm{R} 3}$ is on. Output voltages $\left(V_{\text {out } 1}, V_{\text {out } 2}\right)$, which had been precharged to $V_{D D}$, start to discharge with different discharging rates depending on the corresponding input voltage $V_{i n 1}$ and $V_{i n 2}$. Assuming the case where $V_{i n 2}>$ $V_{\text {in } 1}, V_{\text {out } 2}$ discharges faster than $V_{\text {out } 1}$, hence when $V_{\text {out } 2}$ (discharged by transistor $M_{2}$ drain current), falls down to $V_{D D^{-}}\left|V_{t h p}\right|$ before $V_{\text {out } 1}$ (discharged by transistor $M_{1}$ drain current), the corresponding transistor $M_{L 2}$ will turn on initiating the latch regeneration caused by back-to-back inverters $\left(M_{L 1}, M_{L 2}\right.$ and $\left.M_{L 3}, M_{L 4}\right)$. Thus, $V_{\text {out } 1}$ pulls to $V_{D D}$ and $V_{\text {out } 2}$ discharges to ground. If $V_{i n 2}<V_{i n 1}$, the circuits works vice versa. Nevertheless single-tail Dynamic Comparator shows a high kick-back noise, the small mismatch between differential input CMOS transistor, input-offset voltage in precision applications, such as high-resolution ADC large input-offset voltages cannot be tolerated (Shaik and Rajesh, 2013; Dastagiri and Hari Kishore, 2018).

A conventional double-tail comparator is shown in Fig. 3 (Dastagiri and Hari Kishore, 2018; Rajesh et al., 2016). This circuit enables a large current in the latching stage for fast speed and problems in low power supply voltage, offset input common-mode voltage due to its structure can be overcome, but this comparator requires high accuracy timing between CLKA and $C L K B$. Otherwise the desired output may deviate and it results in is increased power dissipation. The operation of this comparator is as follows. During rest phase $(C L K=0), M_{3}$ and $M_{4}$ are on, charge nodes $\mathrm{Np}$ and $\mathrm{Nn}$ to $V_{D D}$ which in turns charges. During evaluation phase $\left(C L K=V_{D D}\right)$, the tail current transistors $M S 1$ and $M S 2$ turns on. $N p$ and $N n$ nodes common mode voltage decreases and one input dependent differential mode voltage to latch stage. The inverters start to regenerate the voltage difference as soon as the common-mode voltage and the $N p$ and $N n$ nodes is no longer as enough for $M C 1$ and $M C 2$ to clamp the outputs to ground. $M C 1$ and $M C 2$ also provide additional shielding between the input and output, which in turn reduces kickback noise.

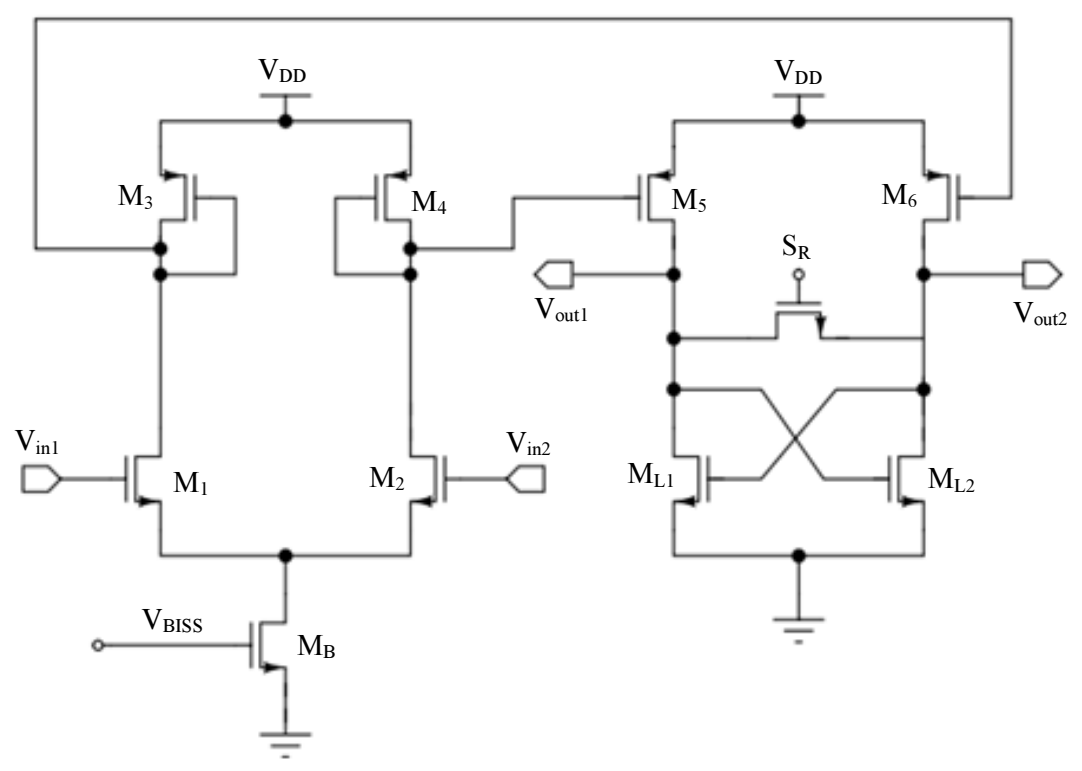

Fig. 1: Pre-amplifier based 


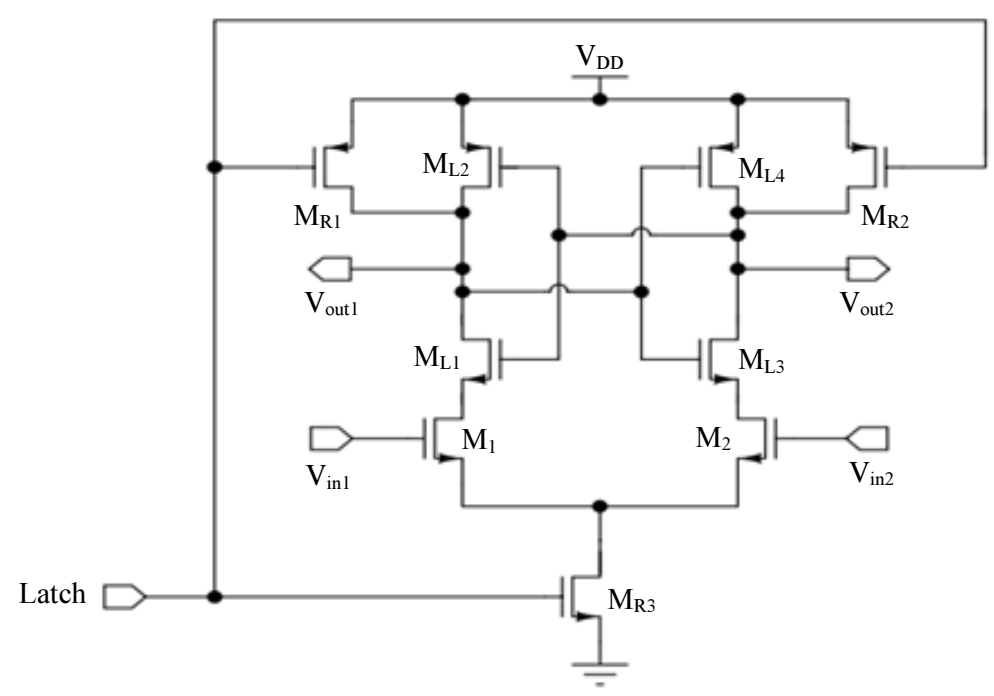

Fig. 2: Conventional dynamic comparator

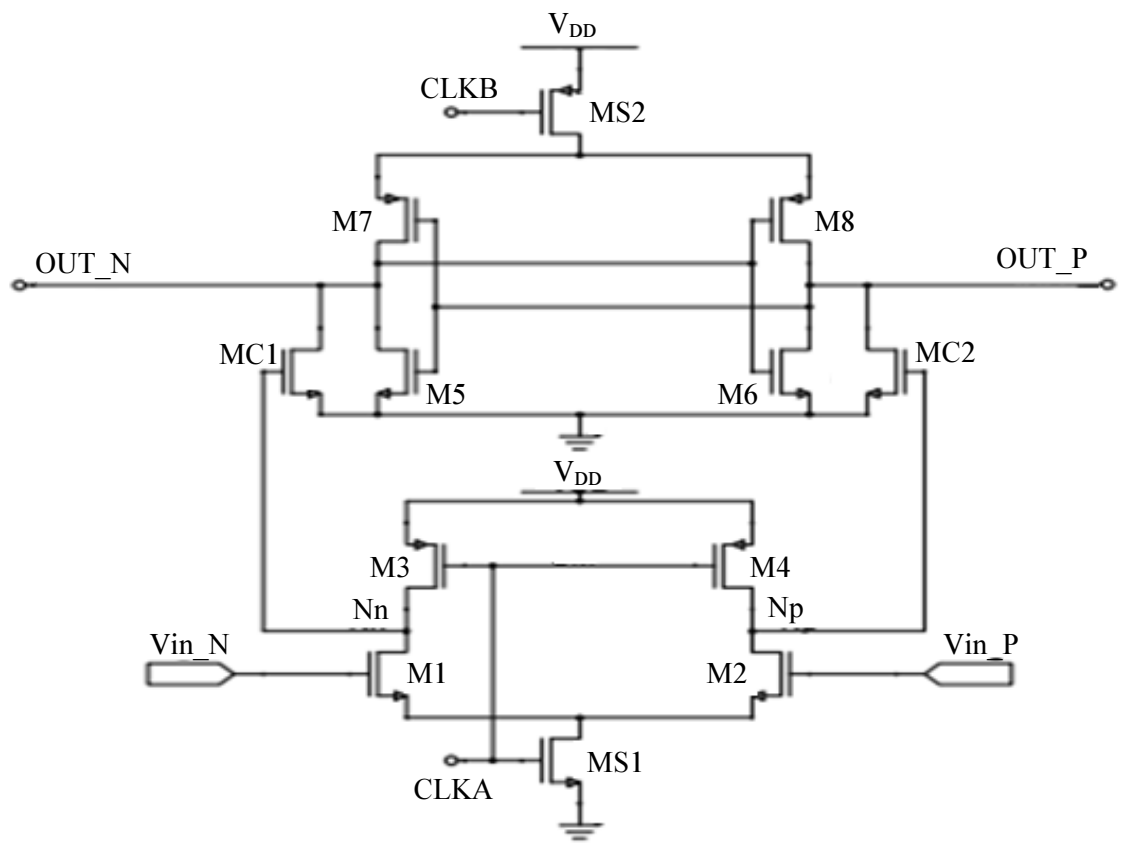

Fig. 3: Conventional double-tail dynamic comparator

\section{Design of the Proposed Dynamic Comparator}

The main idea of proposed comparator isto reduce kickback noise by a capacitive coupling between differential input amplifiers. Figure 4 presented the schematic views of the proposed design. The operation is as follows. During the reset phase (CLK $=0$ ), both PMOS M5 and $M 6$ are turned on and they charge $S N$ and $S P$ nodes capacitance to $V_{D D}$, which turn both transistor $M 7$ and $M 8$ to on and $O n$ and $O p$ nodes capacitance discharge to ground while both PMOS transistor $M 11$ and $M 12$ are being off (no current flows in $M 9$ and $M 10$ cross-coupled). Comparator $O U T_{-} N$ and $O U T_{-} P$ are pulled $M 3-M 4$ to $\mathrm{V}_{\mathrm{DD}}$, MOSFET M13 turn on allow the leakage currents to charge nodes asymmetrically. During the evolution phase (decision-making) when clock signal changes to high state $\left(C L K=V_{D D}\right), M 5$ and $M 6$ are now off while $M S$ is on and the $S N$ and $S P$ nodes capacitance discharged from $V_{D D}$ to ground in a different time rate proportional to the magnitude of each input voltage 
through transistor cross-coupled and differential input amplifier $M 1-M 2$. When $\operatorname{Vin} N$ is large than $V_{i n} P$ (Vin_N $\square$ Vin_P), the current in M3 is larger than the current in M4. $S N$ node drops faster than $S P$ node. The corresponding MOSFETs control: $M 11$ turn on, $M 7$ turn off and M4 turn off breaking the connection to the ground (no current flows in the MOSFETS M12 and $M 2$ ), then $M 12$ turns off. Comparator OUT $N$ node passes to $V_{D D}$. It forces the other $O U T P$ node to ground and M13 turn off. Similarly, when Vin_Nis less than $V i n$ P, the dynamic comparator works in the opposite direction (or vice versa).

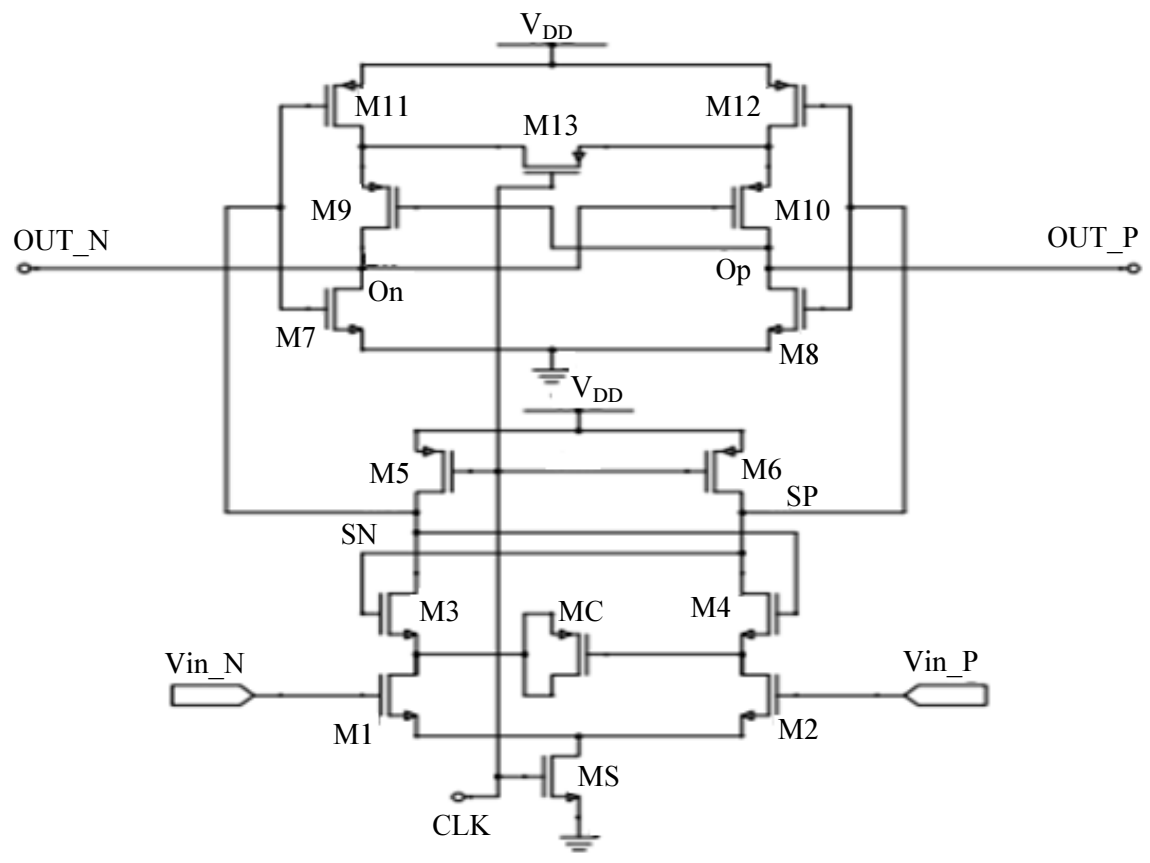

Fig. 4: Proposed dynamic comparator

\section{Schematics of Various Types of Dynamic Latched Comparators}

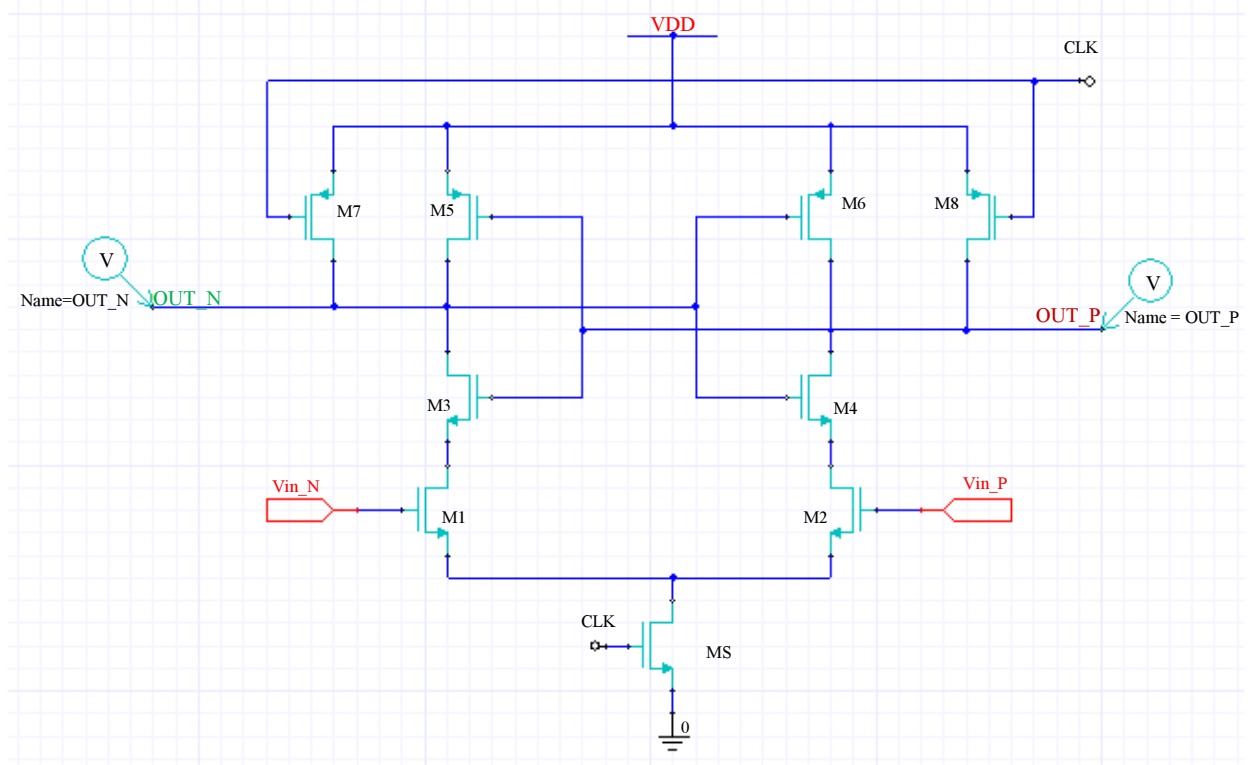

Fig. 5: Conventional dynamic comparator (Comparator 1) 


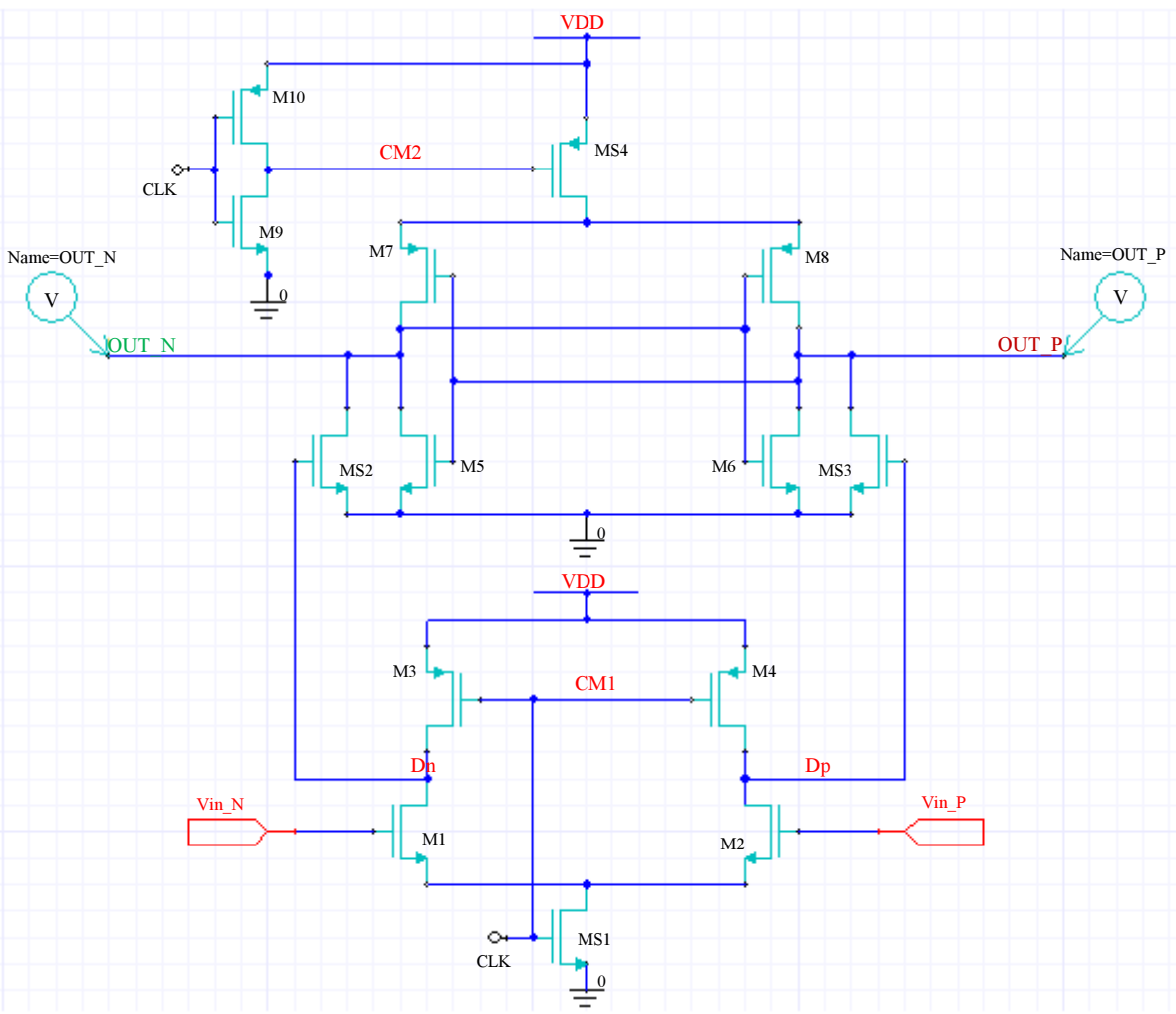

Fig. 6: Double-tail dynamic comparator (Comparator 2)

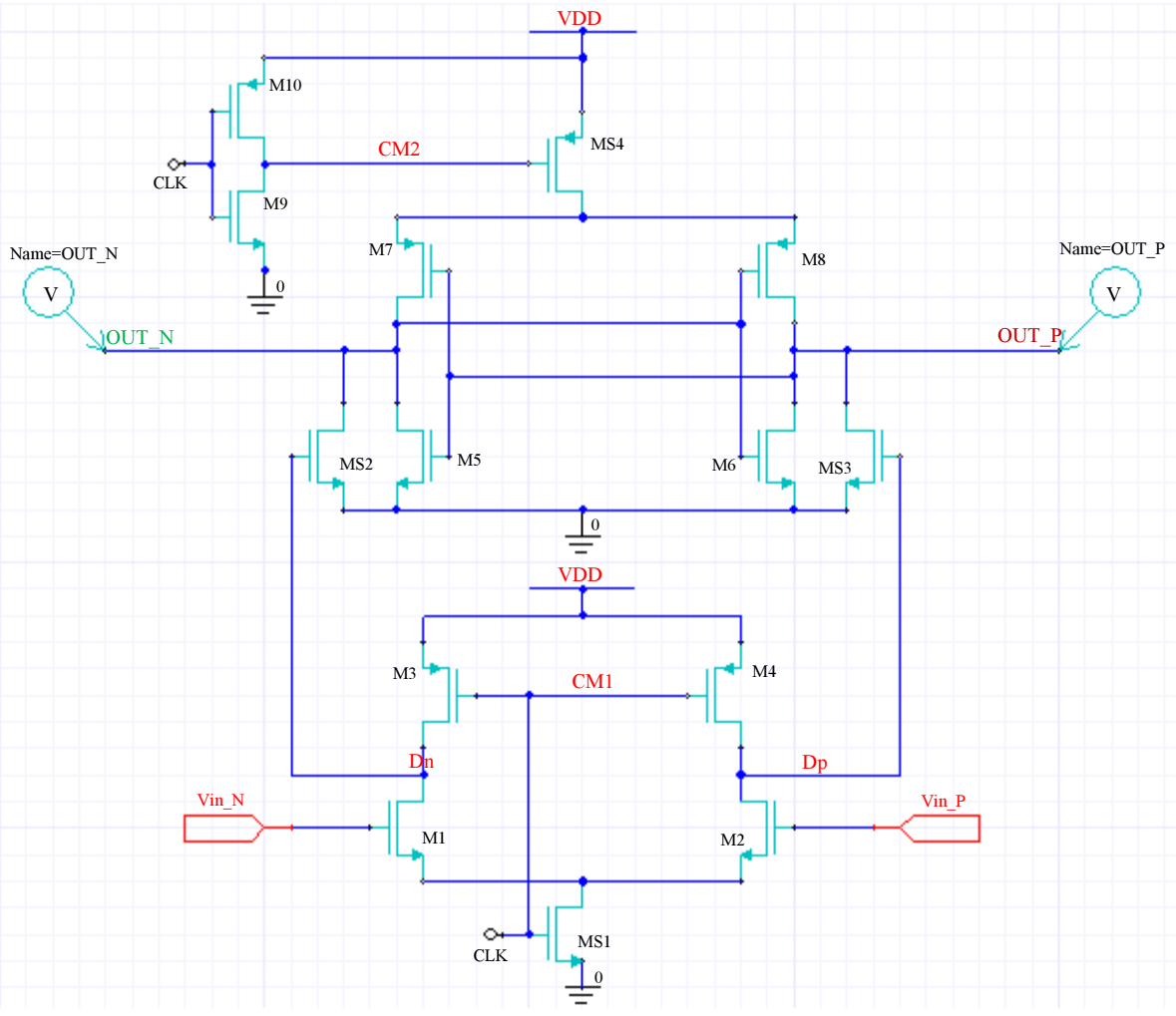

Fig. 7: Dual stage dynamic comparator (Comparator 3) 


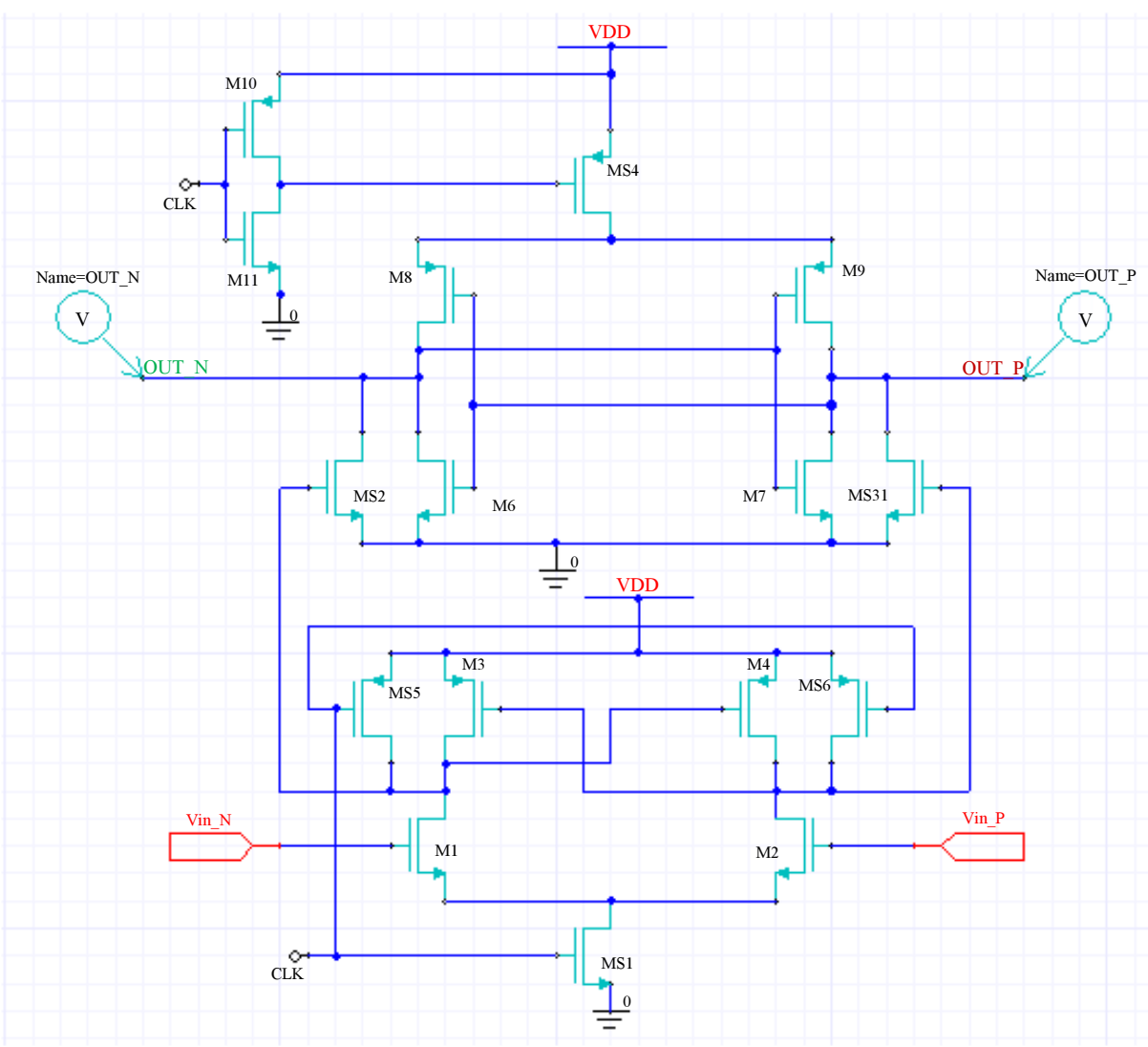

Fig. 8: Proposed dynamic comparator (Comparator 4)

Table 1: Comparison of performance parameters

\begin{tabular}{llllrr}
\hline Comparator type & Number of transistors & Delay $(\mathrm{ps})$ & Power consumption $(\mathrm{mW})$ & Offset $(\mathrm{mV})$ & Kickback noise $(\mathrm{mV})$ \\
\hline Comparator 1 & 09 & 102.00 & 0.0518 & 120.0 & 777.2 \\
Comparator 2 & 14 & 70.00 & 0.1549 & 20.0 & 370.0 \\
Comparator 3 & 16 & 69.11 & 0.1528 & 18.6 & 310.0 \\
Comparator 4 & 15 & 20.23 & 0.1097 & 5.7 & 20.0 \\
\hline
\end{tabular}

Table 2: Comparison of proposed comparator with various supply voltage

\begin{tabular}{lll}
\hline & Technology $90 \mathrm{~nm}$ & \\
Supply voltage & Power dissipation & Delay \\
\hline $1.0 \mathrm{~V}$ & $109.7 \mu \mathrm{W}$ & $20.000 \mathrm{ps}$ \\
$1.2 \mathrm{~V}$ & $135.7 \mu \mathrm{W}$ & $18.225 \mathrm{ps}$ \\
$1.4 \mathrm{~V}$ & $165.0 \mu \mathrm{W}$ & $17.090 \mathrm{ps}$ \\
$1.6 \mathrm{~V}$ & $196.7 \mu \mathrm{W}$ & $16.305 \mathrm{ps}$ \\
\hline
\end{tabular}

\section{Simulation Results and Comparison}

In order to compare the performances of the proposed comparator with the previous works, we designed and simulated each circuit using Ansys Electronic desktop with 90nm PTM technology. For simulation of all designs we used, $V_{D D}(\max )=1 \mathrm{~V}, f_{C L K}(\max )=10 \mathrm{GHz}$, $C_{L O A D}=7 f F$, Temp $=25^{\circ} \mathrm{C}$ and common mode voltage $V_{\text {com }}(\max )=0.45 \mathrm{~V}, \Delta$ Vin $=8 \mathrm{mV}$, fin $=5 \mathrm{Ghz}$.

Finally, simulations of the comparator designs Fig 5 to 8 are done and simulation results are presented including delay, power dissipation, offset and kickback noise shown in Fig. 9 to 12, respectively. Through simulation, we have obtained parameters of the comparator like power dissipation, offset, kickback noise and time delay. Both existing designs and proposed design is simulated, their results are compared and values are tabulated in Table 1, which show the low power dissipation, low time delay, low offset and low kickback noise parameters as it is required for robustness of the ADCs. Furthermore, when we use the variation of power supply for proposed comparator, the results obtain show the improvement of the time delay and the increasing of power dissipation for the bigger power supply, Table 2 . 
:
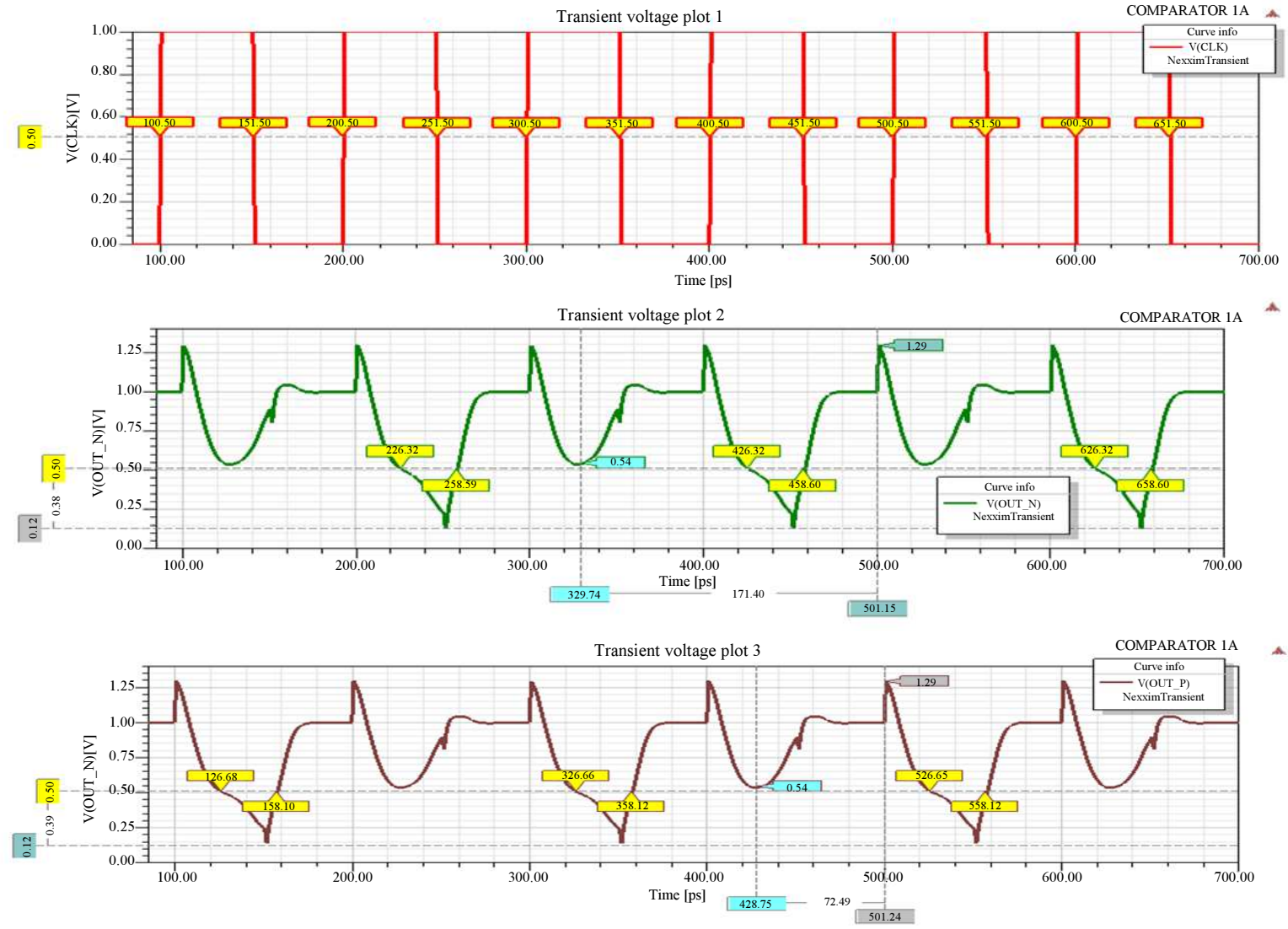

Fig. 9: Simulated waveform of Conventional Dynamic Comparator

:
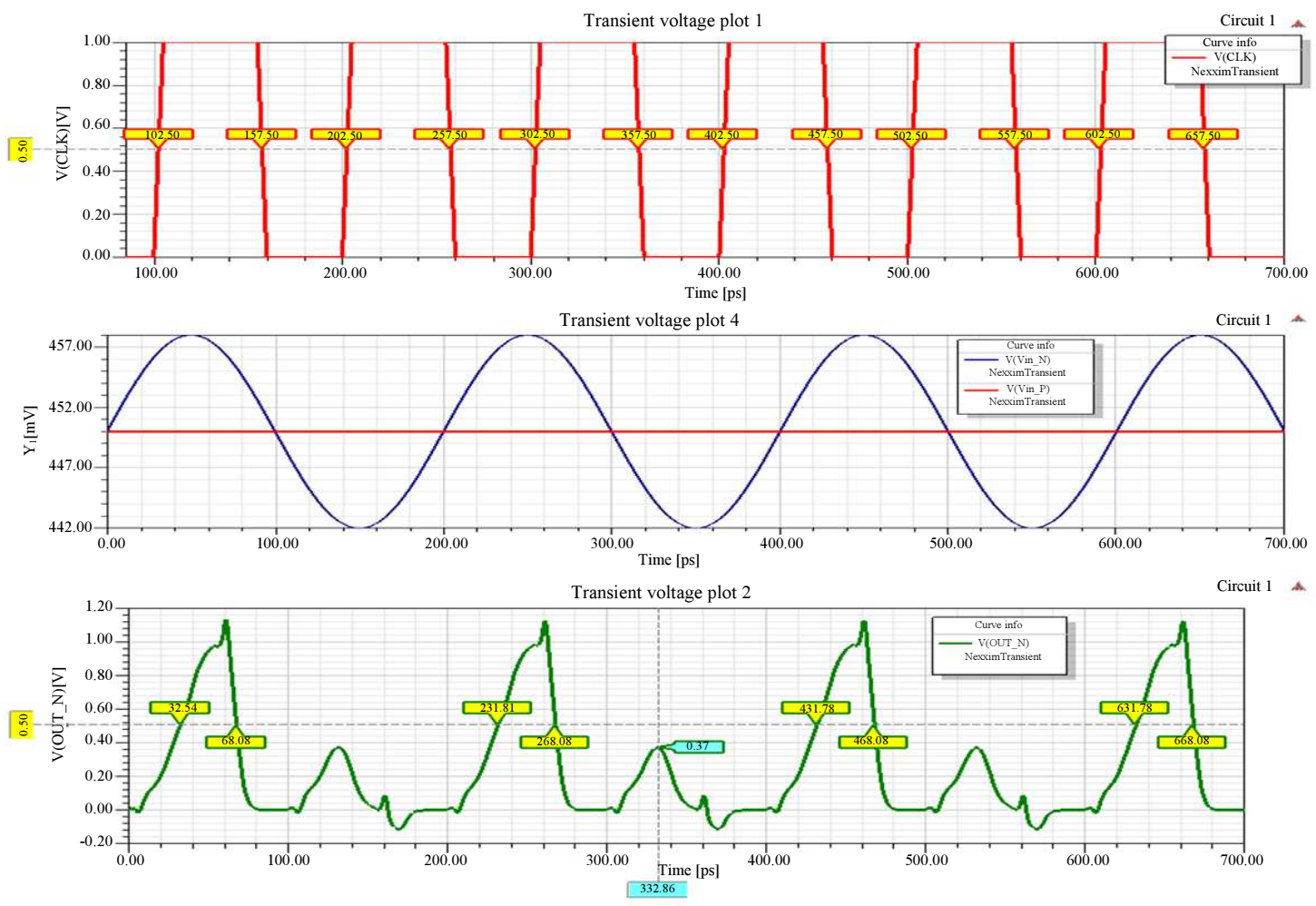


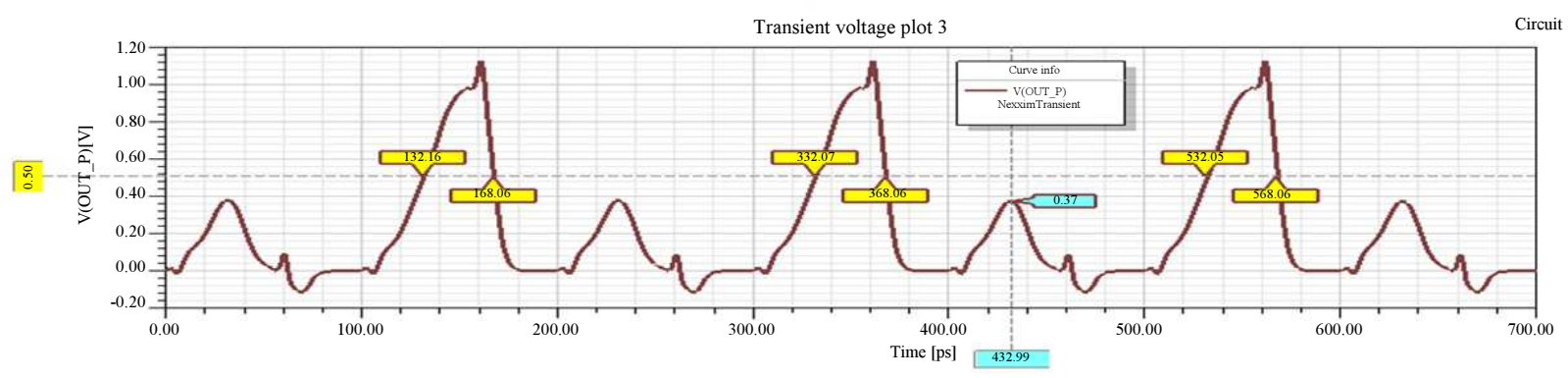

Fig. 10: Simulated waveform of double-tail dynamic comparator
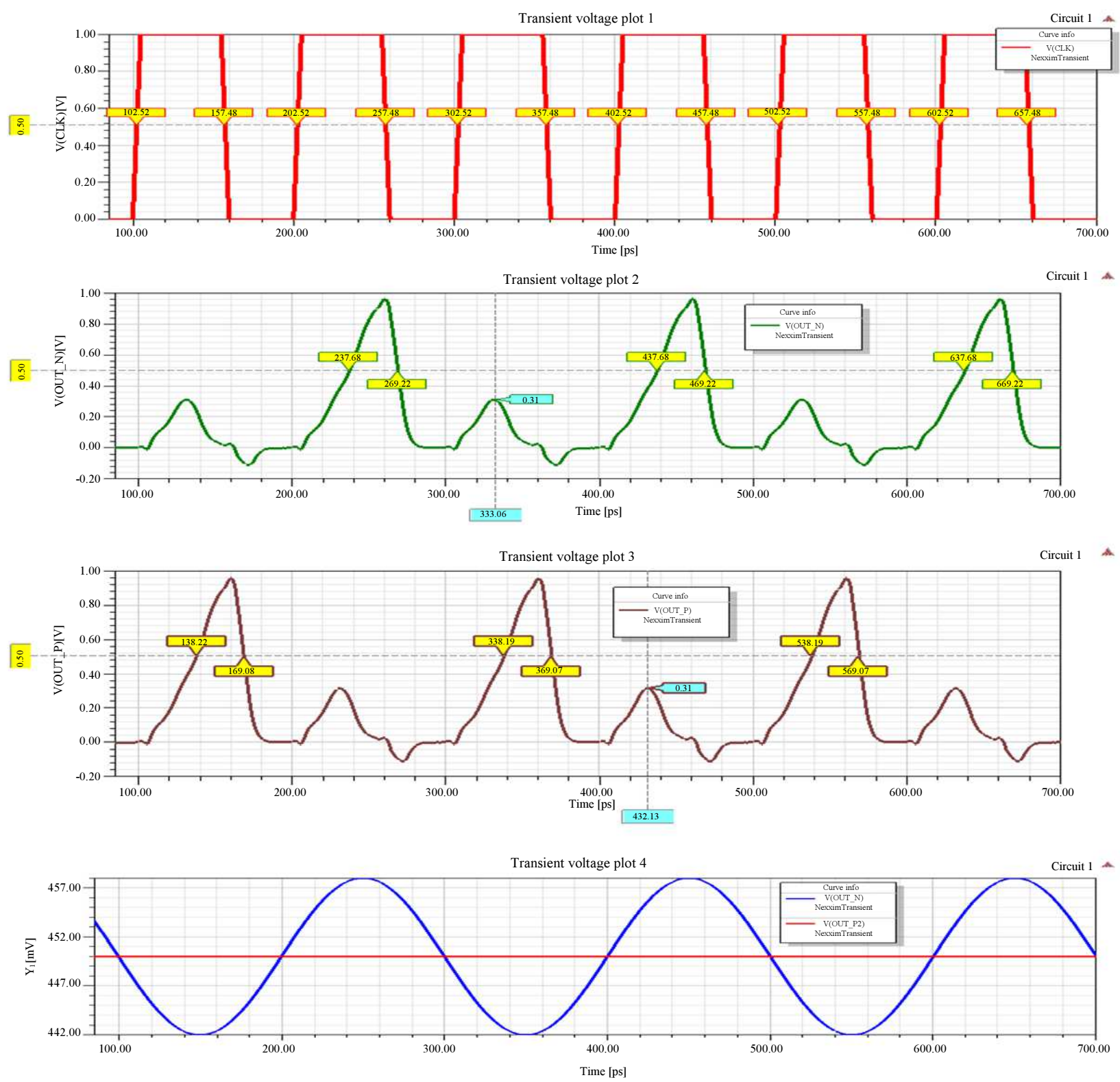

Fig. 11: Simulated waveform of dual stage dynamic comparators 

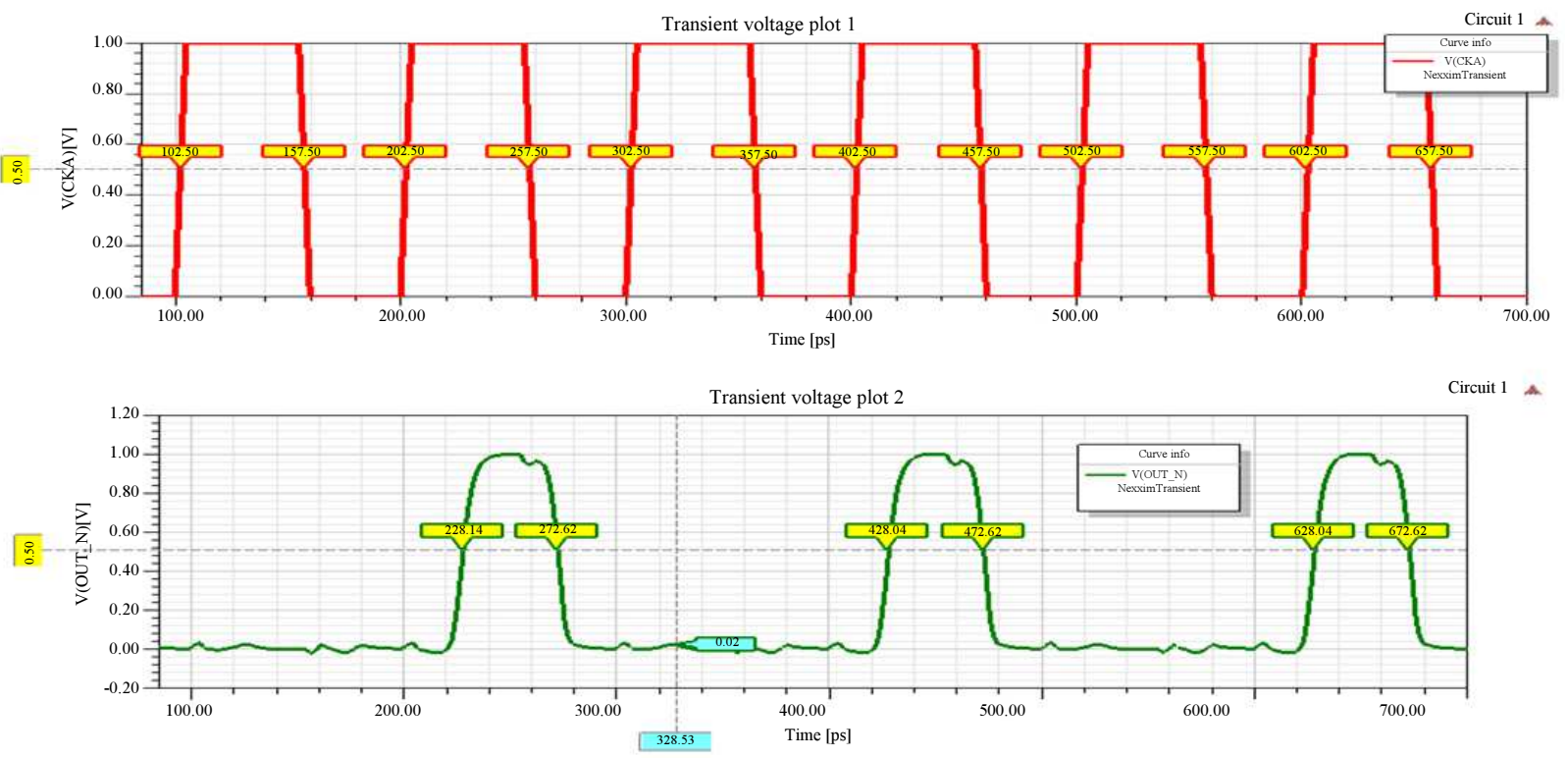

Circuit 1 A
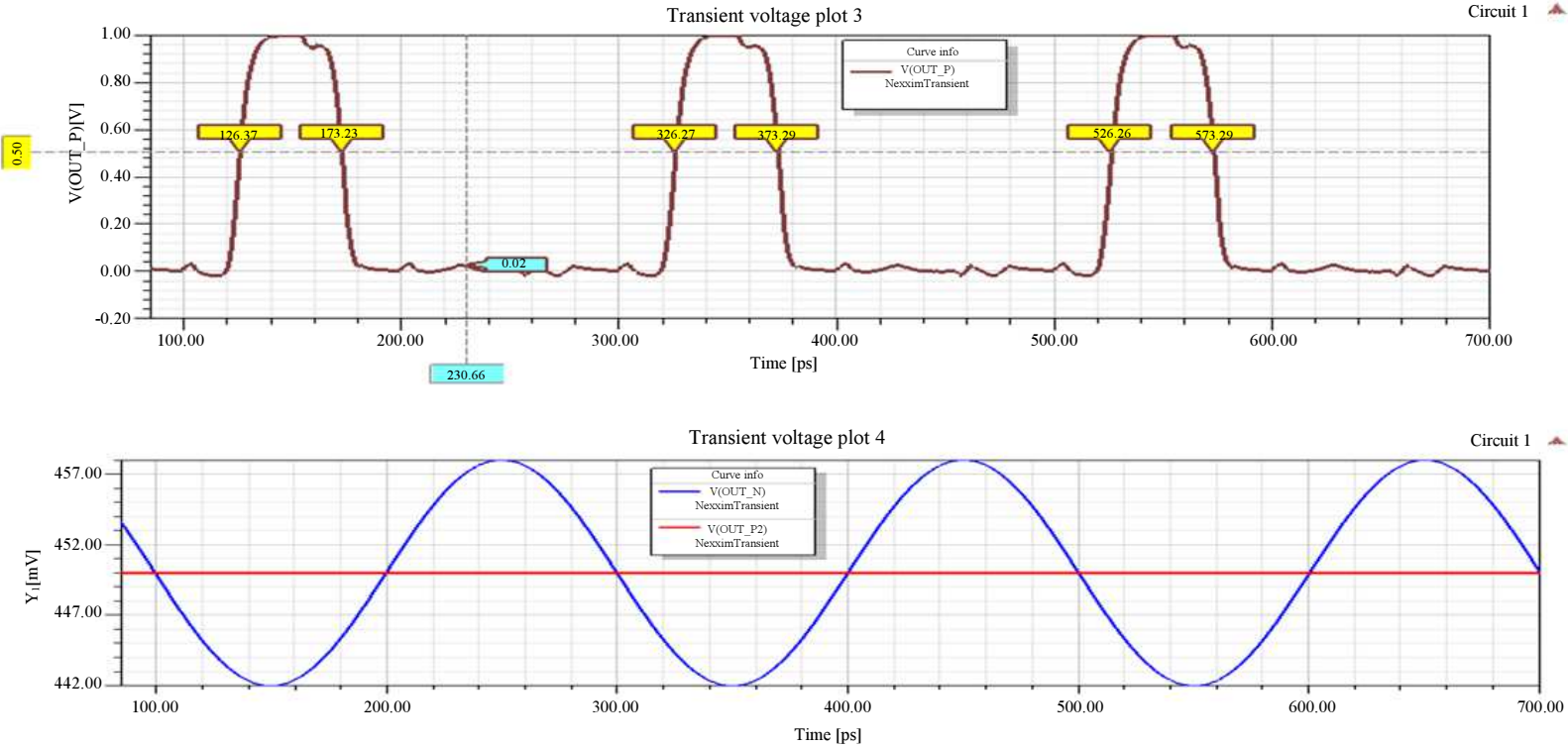

Fig. 12: Simulated waveform of proposed dynamic comparator

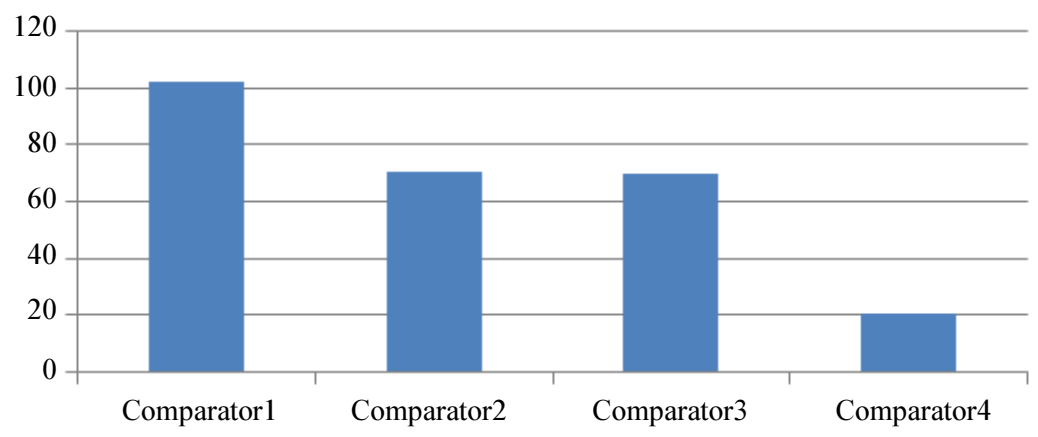

Fig. 13: Delay comparison of dynamic comparators 


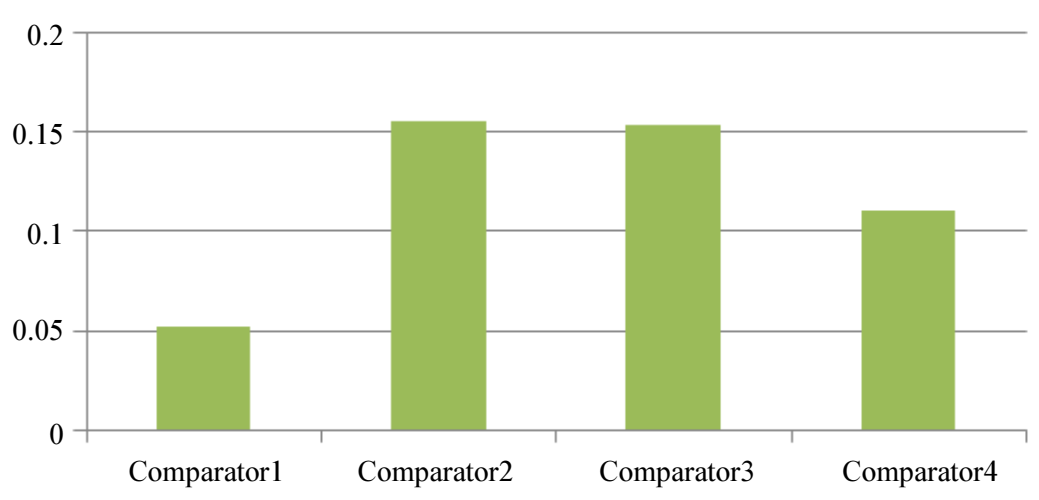

Fig. 14: Power consumption comparison of dynamic comparators

The delay (in ps) comparison of dynamic comparator designs are shown in Fig. 13 in $90 \mathrm{~nm}$ technologies.

The power consumption (in $\mathrm{mW}$ ) comparison of dynamic comparator designs are shown in Fig. 14 in 90 $\mathrm{nm}$ technologies.

\section{Conclusion}

In this paper, the comparator circuits for highspeed ADCs have been presented. The conventional dynamic, conventional double tail and proposed dynamic comparators have been simulated with $90 \mathrm{~nm}$ CMOS technology using Ansys Electronic desktop and their performance parameters such as time delay, power dissipation, offset and kickback noise are compared. The proposed dynamic comparator shows minimal time delay $20.23 \mathrm{ps}$, minimal power consumption of $0.1097 \mathrm{~mW}$ and $5.7 \mathrm{mV}$, minimal offset and minimal kickback noise of $20 \mathrm{mVin} 90 \mathrm{~nm}$ PTM CMOS Technology respectively. The proposed comparator is best suitable for low power and highspeed as it is required for robustness of the ADCs.

\section{Author's contributions}

Wembe Tafo Evariste: Project leader, data interpretation and contribute to the writing of the paper.

Bakoune Pierre Hypolite: Design and simulations model. Also, contribute to the writing of the paper.

Moukengue Imano Adolphe: Project leader. Revise and improve the final drafts of the paper.

\section{Ethics}

The corresponding author confirms that all of the other authors have read and approved the manuscript and no ethical issues involved.

\section{References}

Mongre, R. and R.C. Gurjar, 2014. Design of low power and high speed comparator with $0.18 \mu \mathrm{m}$ technology for ADC application. J. Eng. Res. Applic. 4: 146-153.

Shaik, M.V. and P. Rajesh, 2013. A 3GHz low-offset fully dynamic latched comparator for high-speed and low-power ADCs. Int. J. Emerg. Technol. Adv. Eng., 3: 96-102.

Pedro, M. and J.C. Vital, 2006. Kickback noise reduction techniques for CMOS latched comparators. IEEE Trans. Circuits Syst., 53: 541-545. DOI: $10.1109 /$ TCSII.2006.875308

Iniewski, K., 2015. CMOS front-end electronics for radiation sensors. CMOS Emerging Technologies Research Inc. Vancouver, British Columbia, Canada.

Zbigniew, J., 2016. A $10 \mathrm{Gs} / \mathrm{s}$ latched comparator witch dynamic offset cancellation in $28 \mathrm{~nm}$ FD-SOI process. Proceedings of the SPIE 10175, Electron Technology Conference, (ETC' 16), SPIE. DOI: $10.1117 / 12.2263521$

Dastagiri, N. and K. Hari Kishore, 2018. Design of dynamic latched comparator with reduced kickback noise. Int. J. Pure Applied Math., 118: 289-298.

Wicht, B., T. Nirschl and D. Schmitt-Landsiedel, 2004. Yield and speed optimization of a latch-type voltage sense amplifier. IEEE J. Solid-State Circuits, 39: 1148-1158. DOI: 10.1109/JSSC.2004.829399

Kale, S. and R.S. Gamad, 2010. Design of a CMOS comparator for low power and high speed. Int. J. Electronic Eng. Res., 2: 29-34.

Rajesh, P., M. Priyanka, P. Nalini and M. Chandra Mouli, 2016. An optimized low-voltage low-power double tailcomparator for high-speed ADCs. Int. J. Adv. Eng. Technol., 9: 310-320. 